\title{
An activity measure of sleeping and waking behavior'
}

ROBERT A. LEVITT ${ }^{2}$ UNIVERSITY OF FLORIDA

This report (1) describes a movement-sensing device for discriminating between the sleeping and waking states in small mammals and, (2) presents data on the correlation between a criterion of motionlessness and EEG measured sleep cycle for the rat.

Two methods of determining whether an animal is asleep or awake have been in general use; behavioral observation and the EEG. Both of these methods have severe disadvantages, especially for extended periods of recording. The first requires the continuing presence of an observer, who must subjectively judge the animals sleep-waking state; the second requires the continual monitoring of EEG equipment and records. Also, animals must be implanted with permanent electrodes for EEG recording and the animal trails wires during the study.

The movement-sensing apparatus has the advantage that it is inexpensive (relative to the EEG), does not require surgery or the presence of an experimenter, and may be operated continually for long periods of time (the author has used the devices continually for up to two weeks in studies of sleep deprivation; Levitt, in press).

Dillon (1963) used an ultrasonic movement-sensing device developed by Peacock \& Williams (1962) to record sleeping and waking in the rat. Higgins (1964) has built a transistorized version of the Peacock and Williams unit. The system presented here is a modification of Dillon's. A simple scoring system, a pendulum-type calibrator for adjusting sensitivity, and permanent enclosures have been designed and tested. Using these modifications a series of correlated EEG-activity recordings were collected.

\section{Method}

Six male Long-Evans rats were used. Three were 90-100 and three 180-200 days old.

Sleep and waking were determined by a Grass-III-D EEG. Each minute was scored for waking, slow wave sleep (SWS), or paradoxical sleep (PS) using Swisher's criteria (1962). Whichever of these stages was most prevalent was the condition assigned to an individual minute.

Activity was measured with the ultrasonic device developed by Peacock \& Williams (1962) or the Higgins version (1964). These activity devices are intended for use within a one cubic yard area to detect the movement of small mammals. Both systems generate a 40-Kilocycle sine wave which is radiated as ultrasonic sound (nonaudible) into the test area by a transmitter. These waves permeate the test area and are picked up by a receiver. Any movement within this area produces disturbances in the received portion of the wave, activating a readout unit. An Esterline-Angus Operation Recorder, Model AW, was used to record the pulses transmitted from the activity devices.

The magnitude of movement which causes a pulse can be adjusted through a broad range with a sensitivity control. A pendulum-type

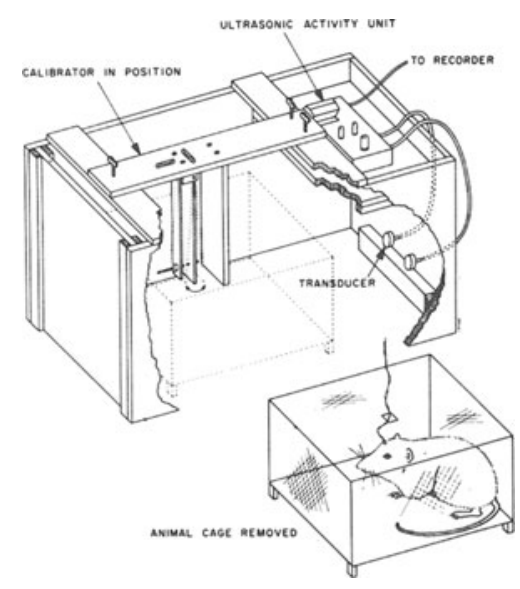

Fig. 1. A view of the movement-sensing system.

calibrator was used to set the sensitivity (Fig. 1). The cage was removed and the pendulum placed on the frame for calibration. The pendulum was then manually held against the swing adjustment at a specified distance from the vertical $(0.20 \mathrm{in}$.), and then released. The time from release until the activity unit stopped responding to the decreasing pendular motion was the unit of calibration.

For EEG-activity recording the animal cage was placed inside an electrically shielded room. The cages were $11 \mathrm{in.} \mathrm{wide,} 16 \mathrm{in.}$ long, and $9 \mathrm{in}$. high. All sides were of wire mesh, permitting the ultrasonic waves to pass through. The cages were individually housed within "wall-board" enclosures measuring 14 in. wide, 32-1/2 in.long, and 16 in. high. This enclosure was designed to bounce the ultrasonic waves throughout the cage, eliminating "dead" spots in the corners and also to prevent leakage of ultra sonic sound into neighboring units, causing interference.

The two activity transducers were placed 3-1/4 in. apart, parallel to, and $10 \mathrm{in.}$ from the cage. The transducers were at a level 3 in. above the cage floor.

Gold bipolar electrodes were implanted under pentobarbital anesthesia. Both electrodes were placed on the same hemisphere, one in the frontal and one in the parietal area. This electrode placement is important, since Swisher (1962) has found it difficult to differentiate PS from waking with other placements.

The scoring rules were developed empirically by taking a correlated sample of EEG-activity data and working out a set of rules that produced a high correlation. The scoring system assigns a number to each minute based on the amount of activity within the four 15-sec. periods making up the minute. Table 1 shows the scoring rules. The point scores for the four 15-sec. periods making up a minute are added together, and the final number defines the activity level for that minute. Using this system a minute can have a score from 0 to 20. Any minute with a total point score from 0 to 3 is considered sleep, and any with a point score from 4 to 20 is waking. There are

Table 1. An Activity Scoring System

(Per 15 sec. observation period)

\begin{tabular}{lcccccc}
$\begin{array}{c}\text { Needle excursions } \\
\text { (pulses) }\end{array}$ & 0 & $1-3$ & $4-8$ & $9-15$ & $16-24$ & 25 or more \\
\begin{tabular}{l} 
Points \\
\hline
\end{tabular} & 0 & 1 & 2 & 3 & 4 & 5 \\
\hline
\end{tabular}


two exceptions to this rule; (1) $1 \mathrm{~min}$. of 3 between $2 \mathrm{~min}$. of 4 or more is scored waking, and (2) $1 \mathrm{~min}$. of 4 between $2 \mathrm{~min}$. of 3 or less is considered sleep.

\section{Resulis and Discussion}

Nineteen $100 \mathrm{~min}$. samples of correlated EEG-activity data were collected at a number of calibration settings ranging from 75 to 215 sec. At all settings between 120 and $200 \mathrm{sec}$. (13 of the 19 samples) the percentage agreement between the EEG and activity measures scored on a minute by minute basis ranged between 86 and 100 per cent; the mean was 93 per cent. The accuracy of the two ultrasonic activity models was not significantly different (Peacock \& Williams, 91\%; Higgins, 94\%), nor was there a significant difference due to the age of the animals (90-100 days old, 92\%; 180-200 days old, 93\%).

Table 2 summarizes the data from this experiment. Only data from the calibration settings between 120 and $200 \mathrm{sec}$. are included. It is interesting to note that the percentage of errors during the three stages is quite similar, suggesting no preponderance of errors of either the sleep-active or waking-nonactive type. Of particular interest is a comparison of the SWS and PS errors. Animal PS is thought to be analogous to human stage I-REM "dream"' sleep (Dement, 1965). Investigators have found muscle tone to be at a minimum during REM or paradoxical sleep (Dement \& Kleitman, 1957; Hall, 1963). In contrast to tonic body movements, both human and animal subjects show a maximum of phasic twitches (fingers, tail, vibrissae) during REM or PS. The data

Table 2 .

Averaged Data on Sleep Cycle and EEG-Activity Correlation

\begin{tabular}{lccc} 
& Waking & $\begin{array}{c}\text { Stoges } \\
\text { SWS }\end{array}$ & PS \\
\hline Total minutes & 796 & 445 & 77 \\
Per cent of time & $61 \%$ & $34 \%$ & $5 \%$ \\
Minutes in error & 57 & 36 & 6 \\
$\begin{array}{l}\text { Per cent of minutes } \\
\text { in error }\end{array}$ & $7.2 \%$ & $8.4 \%$ & $7.8 \%$ \\
\hline
\end{tabular}

from the present experiment would suggest that there is no difference in the total amount of movement during SWS and PS, although a difference in kind may be present.

A total correspondence measure; the percentage agreement between EEG and activity for a 100-min. sample, can be computed. If part of the errors are of the sleep-active type and part of the waking-nonactive type, this measure will be elevated above the minute by minute agreement. This total correspondence measure is important since for many purposes the amount of sleeping and waking during an extended period is the dependent variable of interest. The total correspondence between EEG and activity for the individual 100-min. recording sessions in this experiment averaged 97 per cent.

The ultrasonic activity system should be a useful and reliable means of measuring sleep cycle in small mammals; especially for long term recording. It is realized that it will be necessary to refer to the EEG periodically to recheck the EEG-activity correlation and also to differentiate sleep stages.

\section{References}

Dement, W., \& Kleitman, N. Cyclic variations of EEG during sleep and their relation to eye movements, body motility, and dreaming. EEG clin. Neurophysiol., 1957, 9, 673-690.

Dillon, R. F. The relationship between sleep and activity in the rat. Unpublished master's thesis, University of Florida, 1963. Hall, R. "Activated" sleep in the rat. Science, 1963, 139, 800. Higgins, A. Instruction booklet super-sonic motion detector. Gainesville, Florida: Alton Electronics Co., 1964.

Levitt, R. A. Sleep: deprivation in the rat. Science, in press. Peacock, L. J., \& Williams, M. An ultrasonic device for recording activity, Amer. J. Psychol., 1962, 75, 648-652.

Swisher, J. Manifestations of "activated" sleep in the rat. Science, $1962,138,1110$.

\section{Notes}

1. Supported by an NIH grant (NIH-MH-03881-03) to W. B. Webb and a NASA Traineeship to the author. I thank W, B. Webb and B. N. Bunnell for their advice and criticism during the course of the research and A. E. Fisher for his comments, and criticism of an earlier draft of this manuscript.

2. Now at the University of Pittsburgh. 